\title{
Variability of response times as a marker of diverted attention
}

\author{
Paolo Bartolomeo a,b,*, Eric Siéroff ${ }^{\mathrm{c}}$, Sylvie Chokron ${ }^{\mathrm{d}, \mathrm{e}}$, Caroline Decaix ${ }^{\mathrm{a}, \mathrm{c}}$ \\ a INSERM Unit 324, Centre Paul Broca, 2ter rue d'Alésia, 75014 Paris, France \\ b Neuroscience Department, Henri-Mondor Hospital, Créteil, France \\ ' Université René Descartes (Paris 5), Laboratoire de Psychologie Expérimentale, CNRS URA 8581, Paris, France \\ ${ }^{\mathrm{d}}$ Laboratoire de Psychologie Expérimentale, CNRS UMR 5105, Grenoble, France \\ ${ }^{\mathrm{e}}$ Fondation Ophtalmologique Rothschild, Paris, France
}

Received 30 May 2000; received in revised form 18 September 2000; accepted 28 September 2000

\begin{abstract}
Anderson et al. (Variability not ability: another basis for performance decrements in neglect. Neuropsychologia 2000;38:785796) have recently reported that variability of response times (RTs) progressively increases from the right to the left side in left neglect patients. Anderson et al. propose that this lack of consistency is an important determinant of patients' behaviour, and may result from a deficit independent of other mechanisms causing neglect. Here we suggest that an increase of variability, and not only of RTs, is to be expected when attention is exogenously biased away from the probed location. Consequently, space-based variability can be interpreted in the framework of existing models of unilateral neglect. According to one such model, a basic impairment in left neglect is a bias toward rightward exogenous orienting of attention. As a result, left targets often fail to rapidly capture patients' attention, thus yielding slow RTs. However, since the probability for a left target attracting attention is low but not null, relatively fast RTs can occur on those rare occasions in which a left target does capture patients' attention. The coexistence of these relatively fast with slow RTs could be at the basis of space-based variability in neglect. Empirical support for our hypothesis comes from the results of a re-analysis for variability of cued RTs obtained in 18 normal individuals and six left neglect patients. Cues were peripheral and non-informative, thus eliciting an exogenous attentional shift. For normal individuals, invalid trials yielded less consistent response times than valid trials at short $(150 \mathrm{~ms})$ cue-target interval; for neglect patients, a similar phenomenon occurred for left invalidly-cued targets, thus paralleling the disproportionate cost in RTs typically evoked by this condition in unilateral neglect. We conclude by discussing some possible determinants of gradient-shaped effects and by outlining the implications of space-based variability for current models of unilateral neglect. (C) 2001 Elsevier Science Ltd. All rights reserved.
\end{abstract}

Keywords: Response times; Variability of performance; Spatial attention; Unilateral neglect

\section{Introduction}

For patients suffering from left unilateral neglect, performance on the neglected side can be not only defective, but also extremely variable. Anderson et al. [1] recently argued that such a lack of consistency may be an important determinant of neglect patients' behaviour. Anderson et al. asked five patients with left unilateral neglect and five brain-damaged patients without neglect to press a key in response to the appearance of a target appearing in one of several positions form

* Corresponding author. Tel.: +33-1-40789210; fas: + 33-1$45896848 / 45807293$.

E-mail address: paolo@broca.inserm.fr (P. Bartolomeo). far left to far right on the computer screen. For four of the five neglect patients, reaction times (RTs) progressively increased both in mean duration and in variability from right to left target locations. When only the fastest RTs per position were taken into consideration, these were equally distributed in each spatial position for three neglect patients. The authors concluded that what is impaired in neglect is not necessarily the capacity to respond to events occurring in the neglected hemispace, but rather the ability to produce consistent responses to those events. This study focused on an aspect of neglect performance, variability, that has often been encountered by researchers in the field (see, e.g. $[2,11])$, but has not hitherto been studied in detail. Anderson et al. discounted some possible explanations 
of their findings, such as an effect of hemianopia, a general deficit of sustained attention, a biased ocular exploration, or an interplay between impaired and compensatory systems, and argued for the possibility that 'one mechanism for spatial neglect in some subjects is a spatially modulated effect on sustained performance completely independent of abnormalities in any other cognitive systems' (p. 794).

We propose that space-based variability in unilateral neglect fits existing models of neglect based on a biased exogenous orienting of attention, without the need of postulating additional deficits. Specifically, we suggest that not only longer RTs, but also increased RT variability for events occurring in the neglected space may result from the fact that these stimuli are less likely to attract patients' attention than ipsilesional events. The notion that exogenous orienting is biased toward the right side, but may not be entirely confined to that side, predicts that left-sided stimuli may occasionally capture patients' attention; as a consequence, these stimuli may be processed relatively fast. This could be the basis of the lack of spatial bias in some patients when only 'fast' responses are taken into account (see [1]). If our hypothesis is correct, then measures of RT variability should roughly parallel the distribution of mean or median RTs in neglect patients.

Left neglect patients typically produce the longest RTs when they have to respond to left targets preceded by right-sided, invalid cues $[22,28]$. This RT pattern has been referred to as 'extinction-like' [28], because reminiscent of visual extinction in double simultaneous stimulation. According to our hypothesis, patients' RTs should not only increase, but also show increased variability, for left invalid trials as compared to left valid trials. Furthermore, an increase of variability in RTs should also occur in normal individuals, when their attention is diverted away from the location of the impending target (e.g. by an invalid cue), as compared with situations in which attention is drawn on the target location before target onset (valid trials). If these effects depend on exogenous, rather than endogenous, orienting of attention, they should occur in situations where cues are both peripheral and uninformative about the future location of the target [25], and when the time interval between cue and target (stimulus-onset asynchrony or SOA) is shorter than $300 \mathrm{~ms}$ [24].

We [6] recently obtained data relevant to this issue from 18 normal individuals and six left neglect patients. We employed several variations of the Posner RT paradigm to investigate exogenous and endogenous orienting of attention in unilateral neglect, and found exogenous orienting particularly biased in neglect, whereas endogenous orienting was relatively spared. Here we present a re-analysis for variability of part of these results in order to test our interpretation of Anderson et al.'s [1] findings. We focused on RTs obtained with non-informative, peripheral cues. In these conditions, cues should prompt an exclusively exogenous orienting of attention toward the cued box, especially at short SOAs $[15,24,25]$.

\section{Subjects and method}

\subsection{Subjects}

Six patients with unilateral lesions in the right hemisphere and signs of left neglect, as assessed by a paperand-pencil test battery [3], and 18 age- and education-matched normal individuals took part in the study. All participants were right-handed. Mean age was 59.32 years $($ S.D. $=11.13$, range $45-78$ ) for patients, and 62.83 years (S.D. $=10.19$, range $44-77$ ) for controls. All patients had predominantly retrorolandic vascular lesions, with the exception of one patient, who had suffered from a subcortical frontal lesion as a consequence of a stroke in the territory of the anterior cerebral artery. No patient had hemianopia; all showed visual extinction for left targets on double simultaneous visual stimulation. The mean time elapsed from disease onset was 93.67 days (S.D. $=62.35$, range $22-186$ ).

\subsection{Apparatus and stimuli}

Stimulus presentation and response collection were controlled by the Psychlab software [14]. Three black empty square boxes, with a $10-\mathrm{mm}$ long, 0.34-mm thick side, were displayed on a white background. The boxes were horizontally arranged, the central box being located at the centre of the screen. The central box contained a small black rectangular fixation point $\left(1.02 \times 1.34 \mathrm{~mm}^{2}\right)$. Distance between boxes was $30 \mathrm{~mm}$. Cues consisted of a $300-\mathrm{ms}$ thickening (from 0.34 to $0.68 \mathrm{~mm}$ ) of the contour of one of the lateral boxes. The target was an asterisk $4.40-\mathrm{mm}$ in diameter, appearing inside one of the lateral boxes.

\subsection{Procedure}

Participants sat in front of a computer monitor at a distance of $\sim 50 \mathrm{~cm}$. Each trial began with the appearance of the three placeholder boxes for $500 \mathrm{~ms}$. Then the cue followed during $300 \mathrm{~ms}$. The target appeared at a variable stimulus-onset asynchrony (SOA; 150, 550 or $1000 \mathrm{~ms}$ ) from the cue, and remained visible until a response was made, in order to minimize the possibility of omissions. Participants were instructed to maintain fixation on the fixation point and to respond to the target as quickly and accurately as possible, by pressing the centre of the space bar with their right index finger. Eye movements were observed by one of the experimenters. After an intertrial interval of $1000 \mathrm{~ms}$, a new 

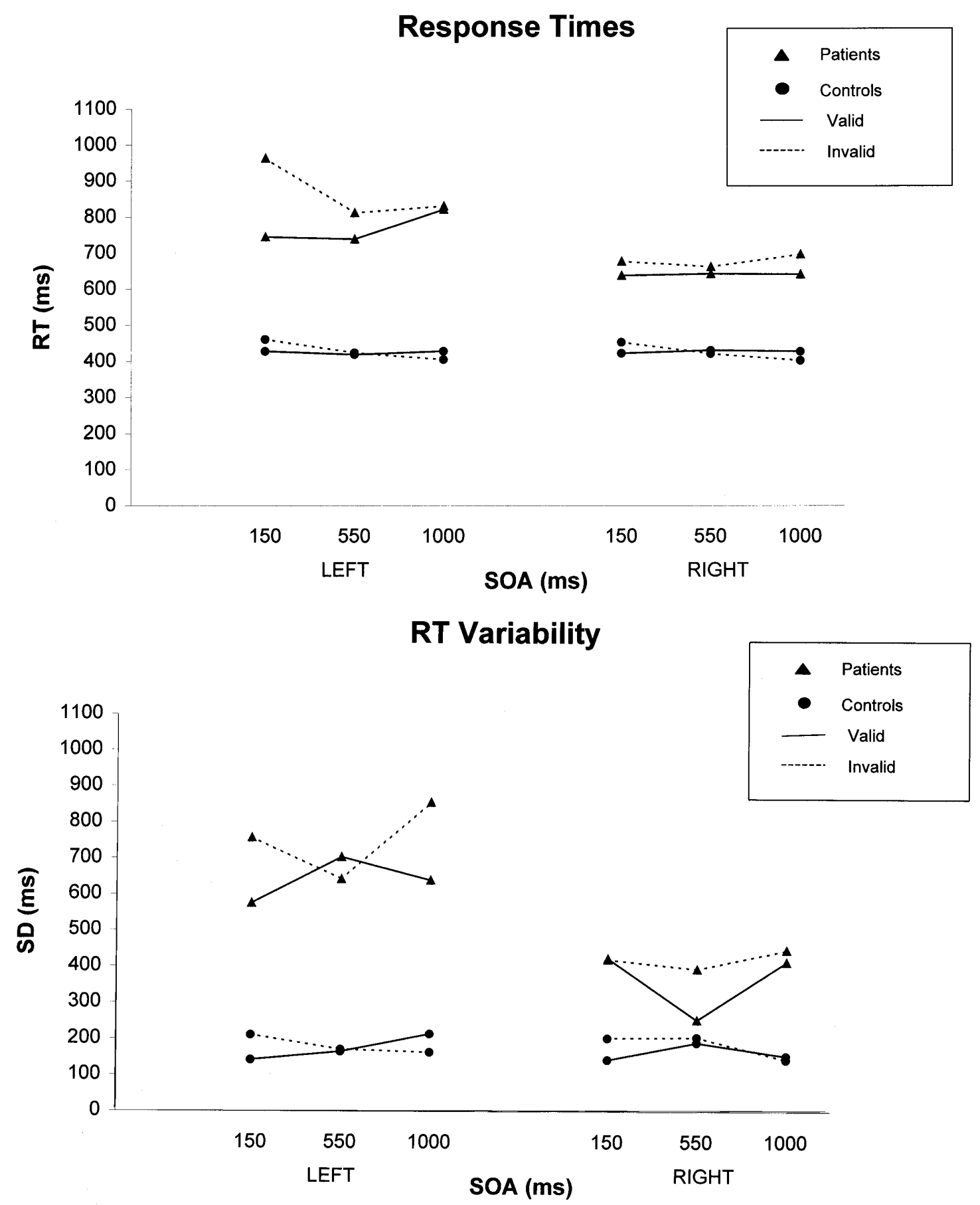

Fig. 1. Response times (upper panel) and their within-subjects variability (lower panel) for neglect patients (triangles) and normal participants (circles). Solid lines, valid condition; dashed lines, invalid condition. SOA, stimulus-onset asynchrony.

trial began. Participants were informed that the target could appear in the cued or in the uncued box with equal probabilities. Each experiment consisted of two blocks of 150 trials preceded by 30 practice trials. A brief period of rest was allowed between blocks. Only RTs in the range of $150-5000 \mathrm{~ms}$ were entered into analysis. For each participant, the median RT and the standard deviation (S.D.) of the mean were calculated and entered into separate repeated-measures analyses of variance (ANOVAs) with group (patient, control) as between factor and side (left, right), cue (valid, invalid), and SOA (150, 550, $1000 \mathrm{~ms})$ as within factors.

\section{Results}

Response times and their within-subjects variability (as measured by the S.D. of the mean) are presented in 
Fig. 1. Results for median RTs are fully presented and discussed elsewhere [6]. To summarise, neglect patients had slower RTs than controls. Controls showed an advantage of valid over invalid trials at $150-\mathrm{ms}$ SOA; for longer SOAs, this advantage turned into a cost, as predicted by the notion of inhibition of return $[20,26,27,29]$. Neglect patients showed a disproportionate cost for left targets preceded by right (invalid) cues; this cost was maximal at the shortest SOA, consistent with the idea of a biased exogenous orienting in neglect. No significant validity effect emerged for right targets, probably because patients tended to ignore the $300-\mathrm{ms}$ cues presented on the left side, and right-sided valid cues could give no further advantage to right targets, which already tended to capture patients' attention.

Most relevant for our present purposes was the ANOVA conducted on S.D.s. Neglect patients had less consistent RTs (mean S.D., $546 \mathrm{~ms}$ ) than controls (170 $\mathrm{ms}), F(1,22)=35.68, P<0.0001$. There was an effect of side, $F(1)=10.98, P<0.005$, which interacted with the group, $F(1,22)=8.60, P<0.01$. The source of the interaction was the fact that in neglect patients the variability for left targets $(697 \mathrm{~ms})$ was much greater than that for right targets (394 ms), whereas in controls there was no such asymmetry (left: $174 \mathrm{~ms}$; right: 160 $\mathrm{ms})$. The type of cue had an effect, $F(1)=5.97, P<$ 0.05 , which again interacted with the group, $F(1,22)=$ 5.91, $P<0.05$, because for patients there was greater variability for invalid trials $(588 \mathrm{~ms})$ than for valid trials $(504 \mathrm{~ms})$, while there was no such effect in controls (170 ms for both conditions). Finally, the four-way interaction between side, SOA, cue and group was also significant, $F(2,44)=3.77, P<0.05$. Paired comparisons revealed that, for controls, invalid trials at 150-ms SOA yielded more variable RTs (S.D., $209 \mathrm{~ms}$ ) than valid trials $(144 \mathrm{~ms}), F(1)=6.86, P<0.05$. For longer SOAs, this cue validity effect reverted to a tendency for invalid trials to evoke less variable RTs than valid trials, $F(1)=3.36, P=0.07$, a tendency reminiscent of the phenomenon of inhibition of return. When neglect patients responded to left targets, at $150-\mathrm{ms}$ SOA the difference in variability between invalid trials $(758 \mathrm{~ms})$ and valid trials $(578 \mathrm{~ms})$ was marginally significant, $F(1)=4.14, P=0.055$. Patients' RTs to left targets were more variable for invalid than for valid trials also at 1000-ms SOA (valid: $641 \mathrm{~ms}$; invalid: $857 \mathrm{~ms}), F(1)=5.91, P<0.05$. No significant validity effect emerged for right targets, similarly to results for median RTs.

\section{Discussion}

In the literature on normal attention, the effects of orienting on performance have been analysed mainly in terms of accuracy and mean or median RTs. Within- subjects variability, on the other hand, has been largely ignored. However, an effect of attention on variability is to be expected, because, on the one hand, in normal individuals the mean and the S.D. of response times covary as a function of the intensity of the stimulus [18]; mean RTs and their S.D.s increase in parallel as stimulus intensity decreases. On the other hand, spatial attention is known to enhance perceptual sensitivity at the attended location [7,12,23]. If this enhancement is interpreted as a subjective increase in stimulus intensity, one can conclude that not only mean RTs, but also their variability should increase at unattended locations.

Consistent with these ideas, our results demonstrate that, for normal individuals, variability of cued RTs (as measured by their S.D.s) is significantly greater for invalid than for valid trials in a situation in which cues evoke an exogenous orienting of attention (non-informative peripheral cues at 150 -ms SOA). This result parallels the pattern of median RTs. Neglect patients showed a generally less consistent performance than normal controls, thus confirming the Anderson et al.'s [1] results. According to our hypothesis, this increased variability may depend on the fact that, in unilateral neglect, the attentional capture exerted by visual stimuli is deficient not only for the left, neglected side, but also for the right side, albeit to a lesser extent [4]. Again consistent with Anderson et al.'s findings, neglect patients' variability of RTs was maximal for left target. In addition, we showed that variability was a prominent feature of responses to left invalidly cued targets, i.e. the very same condition that evokes the phenomenon of extinction-like RT pattern [28], an effect whose size is proportional to the amount of left neglect [22]. The one exception to the general parallelism between the amount and the variability of RTs was that neglect patients showed more variability for invalidly than for validly cued left targets also at 1000-ms SOA. In this condition, valid and invalid trials yielded similar RTs (see Fig. 1, upper panel). To account for the absence of an extinction-like RT pattern at 1000-ms SOA, one could speculate that patients, who were aware of the non-informative value of cues, tried to endogenously spread their attention over the entire stimulus array after cue onset (endogenous processes develop slowly, thus resulting most evident at long SOAs). If so, a conflict could have arisen between endogenous orienting and the exogenous capture exerted by the right, invalid cue. This conflict might have produced increased variability of RTs with respect to valid trials. Independent evidence [6] does suggest that endogenous orienting capacities are preserved, if slowed, in neglect patients.

Our results offer the possibility of interpreting spacebased variability of performance in the framework of existing models of unilateral neglect, without the need 
of postulating additional impairments. If neglect patients suffer from a biased orienting of attention, which concerns primarily exogenous shifts $[2,11,13]$, then it is conceivable that visual stimuli occurring from the right to the left side be progressively less likely to capture patients' attention. This would provoke, on the one hand, an increase in detection time for left-sided stimuli as compared to right-sided ones; on the other hand, variability of RTs would be spatially affected, with occasional fast responses to left targets on those rare occasions in which they rapidly captured patients' attention ${ }^{1}$.

Also the finding that the amount of variability follows a horizontal gradient, with S.D.s increasing form right to left, can be accounted for by existing models of neglect. For example, Kinsbourne has proposed that 'attention is directed along a vector resulting from the interaction of paired opponent processors that are controlled by the right and left hemispheres respectively, each of which directs attention toward the opposite end of a visual display. Unilateral neglect results from an imbalance between these opponent processors, such that the undamaged one is dishinibited..., biasing attention towards the opposite side of arrays that are laterally extended in space' ([17], p. 63f) ${ }^{2}$. The notion of a gradient stems from neglect patients' tendency to orient toward the rightmost stimulus of the visual scene, in relative independence of the stimulus position with respect to the midline (see, e.g. $[10,19])$. The probabilistic nature of the gradient implies that not only error rates and response times, but also variability progressively increase from the right to the left. In Kinsbourne's words, ' $[t]$ here is no point on the gradient at which the probability of detecting a target is zero' ([17], p. 64). It is thus conceivable that even stimuli arising in usually neglected portions of space may occasionally yield fast RTs. This possibility can account for the finding that, at least in some patients, the distribution of these fast RTs shows no relation to horizontal target location [1]. Alternatively, gradient-shaped findings such as those obtained by Anderson et al. [1] may result from sequential dependency of each trial on the prior ones. Though sequential dependency in neglect has not been extensively explored, it is plausible that a (relatively) right-

\footnotetext{
${ }^{1}$ Since the response to all the possible events involved in the Anderson et al.'s study, as well as in ours, is always the same (press the spacebar), whereas the events' locations are different, it seems logical to conclude that any observed difference in performance depends on event detection rather than on response production per se.

${ }^{2}$ See, however, Ref. [4] for caution against too a literal acceptation of the notion of left neglect as resulting from pathologically enhanced attention toward the right. Across different patients, not only RTs to left targets, but also RTs to right targets progressively increase with increasing neglect; this suggests that the rightward attentional bias in neglect is one of defective, and not enhanced, attention.
}

sided stimulus produces longer RTs for a subsequent target on its left (see [28]); on the other hand, a right target may shorten the response time for a subsequent target occurring at the same spatial position [5]. In an experimental situation in which targets can occur at several horizontally arranged positions, the more a target occurs leftward, the more likely it has been preceded by a target at its right. As a consequence of sequential dependency, then, average RTs may progressively increase from the right to the left. For left targets, however, relatively fast RTs will always be possible in those rare instances in which these targets were preceded by targets either at the same spatial location, or to the left of it. Anderson et al. [1] refuted this possibility by showing that, for two of their patients, a left-right gradient in variability was still present when considering only the targets preceded by a target on their left. However, the slope of the regression line seems less steep for left-preceding than for rightpreceding trials (see Fig. 3 in Ref. [1]), thus suggesting a possible participation of sequential dependency in the gradient effect. This idea could be tested by considering whether the rare left targets that evoked fast RTs consistently followed another left target; such a finding would support the sequential dependency account.

The phenomenon of space-based variability has implications for current models of left unilateral neglect. As one of us [2] had already pointed out, it does not agree with models based on a rightward shift of a reference frame for space exploration (see, e.g. [16]), which would predict, rather, impaired but stable performance for left targets. Also models based on an amputation [8] or on a distortion [9,21] of a mental representation of space seem unlikely to readily explain such variability. A distorted spatial representation, for example, would be expected to impair performance on the left side, but it should not increase variability, because stimuli arising in a given location would always fall in the same place on the (distorted) representational medium, thus presumably always evoking the same response (unless one makes the ad hoc assumption that the distortion of spatial coordinates may be variable in time). The impressive parallelism shown here between normal individuals and neglect patients, with both groups demonstrating increased variability of response times when attention is diverted exogenously away from the probed location, appears instead to support models of neglect referring to a biased exogenous orienting of attention in space.

\section{Acknowledgements}

We wish to thank Dr Britt Anderson and three anonymous reviewers for helpful comments and suggestions, and Dr Anne Petrov for reviewing the English. 


\section{References}

[1] Anderson B, Mennemeier M, Chatterjee A. Variability not ability: another basis for performance decrements in neglect. Neuropsychologia 2000;38:785-96.

[2] Bartolomeo P. The novelty effect in recovered hemineglect. Cortex 1997;33:323-32.

[3] Bartolomeo P, Chokron S. Egocentric frame of reference: its role in spatial bias after right hemisphere lesions. Neuropsychologia 1999;37:881-94

[4] Bartolomeo P, Chokron S. Left unilateral neglect or right hyperattention? Neurology 1999;53:2023-7.

[5] Bartolomeo P, Chokron S, Siéroff E. Facilitation instead of inhibition for repeated right-sided events in left neglect. NeuroReport 1999;10:3353-7.

[6] Bartolomeo P, Siéroff E, Decaix C, Chokron S. Modulating the attentional bias in unilateral neglect: the effects of the strategic set. Experimental Brain Research, in press.

[7] Bashinski HS, Bacharach VR. Enhancement of perceptual sensitivity as the result of selectively attending to spatial locations. Perception and Psychophysics 1980;28:241-8.

[8] Bisiach E, Capitani E, Luzzatti C, Perani D. Brain and conscious representation of outside reality. Neuropsychologia 1981;19:543-51.

[9] Bisiach E, Pizzamiglio L, Nico D, Antonucci G. Beyond unilateral neglect. Brain 1996;119:851-7.

[10] De Renzi E, Gentilini M, Faglioni P, Barbieri C. Attentional shifts toward the rightmost stimuli in patients with left visual neglect. Cortex 1989;25:231-7.

[11] D'Erme P, Robertson I, Bartolomeo P, Daniele A, Gainotti G. Early rightwards orienting of attention on simple reaction time performance in patients with left-sided neglect. Neuropsychologia 1992;30:989-1000.

[12] Downing CJ. Expectancy and visual-spatial attention: effects on perceptual quality. Journal of Experimental Psychology: Human Perception and Performance 1988;14:188-202.

[13] Gainotti G, D'Erme P, Bartolomeo P. Early orientation of attention toward the half space ipsilateral to the lesion in patients with unilateral brain damage. Journal of Neurology, Neurosurgery and Psychiatry 1991;54:1082-9.

[14] Gum, T. Psychlab software, 1996.

[15] Jonides J. Voluntary versus automatic control over the mind's eye's movement. In: Long J, Baddeley A, editors. Attention and Performance XI. Hillsdale, NJ: Lawrence Erlbaum, 1981:187283.
[16] Karnath H-O. Disturbed coordinate transformation in the neural representation of space as the crucial mechanism leading to neglect. Neuropsychological Rehabilitation 1994:4:147-50.

[17] Kinsbourne M. Orientational bias model of unilateral neglect: evidence from attentional gradients within hemispace. In: Robertson IH, Marshall JC, editors. Unilateral Neglect: Clinical and Experimental Studies. Hove, UK: Lawrence Erlbaum, 1993:63-86.

[18] Luce RD. Response Times: Their Role in Inferring Elementary Mental Organization. New York: Oxford University Press, 1986.

[19] Marshall JC, Halligan PW. Does the midsagittal plane play any privileged role in 'left' neglect? Cognitive Neuropsychology 1989;6:403-22.

[20] Maylor EA, Hockey R. Inhibitory component of externally controlled covert orienting in visual space. Journal of Experimental Psychology: Human Perception and Performance 1985;11:777-87.

[21] Milner AD, Harvey M. Distortion of size perception in visuospatial neglect. Current Biology 1995;5:85-9.

[22] Morrow LA, Ratcliff G. The disengagement of covert attention and the neglect syndrome. Psychobiology 1988;16:261-9.

[23] Müller HJ, Findlay JM. Sensitivity and criterion effects in the spatial cuing of visual attention. Perception and Psychophysics 1987:42:383-99.

[24] Müller HJ, Findlay JM. The effect of visual attention on peripheral discrimination thresholds in single and multiple element displays. Acta Psychologica 1988;69:129-55.

[25] Müller HJ, Rabbitt PM. Reflexive and voluntary orienting of visual attention: time course of activation and resistance to interruption. Journal of Experimental Psychology: Human Perception and Performance 1989;15:315-30.

[26] Posner MI, Cohen Y. Components of visual orienting. In: Bouma H, Bouwhuis D, editors. Attention and Performance X. London: Lawrence Erlbaum, 1984:531-56.

[27] Posner MI, Rafal RD, Choate LS, Vaughan J. Inhibition of return: Neural basis and function. Cognitive Neuropsychology 1985;2:211-28.

[28] Posner MI, Walker JA, Friedrich FJ, Rafal RD. Effects of parietal injury on covert orienting of attention. Journal of Neuroscience 1984;4:1863-74.

[29] Rafal R, Henik A. The neurology of inhibition: Integrating controlled and automatic processes. In: Dagenbach D, Carr TH, editors. Inhibitory Processes in Attention, Memory and Language. San Diego, CA: Academic Press, 1994:1-51. 